This item was submitted to Loughborough's Research Repository by the author.

Items in Figshare are protected by copyright, with all rights reserved, unless otherwise indicated.

\title{
Nonlinear model predictive coordinated standoff tracking of a moving ground vehicle
}

PLEASE CITE THE PUBLISHED VERSION

http://dx.doi.org/10.2514/1.56254

\section{PUBLISHER}

(c) American Institute of Aeronautics and Astronautics

\section{VERSION}

AM (Accepted Manuscript)

\section{PUBLISHER STATEMENT}

This work is made available according to the conditions of the Creative Commons Attribution-NonCommercialNoDerivatives 4.0 International (CC BY-NC-ND 4.0) licence. Full details of this licence are available at: https://creativecommons.org/licenses/by-nc-nd/4.0/

\section{LICENCE}

CC BY-NC-ND 4.0

\section{REPOSITORY RECORD}

Kim, Seungkeun, Hyondong Oh, and Antonios Tsourdos. 2015. "Nonlinear Model Predictive Coordinated Standoff Tracking of a Moving Ground Vehicle”. figshare. https://hdl.handle.net/2134/17841. 


\title{
Nonlinear Model Predictive Coordinated Standoff Tracking of Moving Ground Vehicle*
}

\author{
Seungkeun $\mathrm{Kim}^{\dagger}$, Hyondong $\mathrm{Oh}^{\ddagger}$, and Antonios Tsourdos $§$ \\ Cranfield University, Defence Academy of the UK, Swindon, SN6 8LA, United Kingdom
}

This paper proposes a nonlinear model predictive control framework for coordinated standoff tracking by a pair of unmanned aerial vehicles. The benefit of this approach is to get optimal performance compared to using a decoupled controller structure: heading control for standoff-distance keeping and speed control for phase-keeping. The overall controller structure is fully decentralized in a fact that each unmanned aerial vehicle optimizes its controller based solely on the future propagation of the pair vehicle states and the target estimates received via communication. This paper uses an acceleration model for a sophisticated and realistic target dynamics, which can consider more reasonable system noise covariance matrix reflecting the target's motion characteristics. To simplify optimization formulation and decrease computation burden, a new manipulation using inner product of position vectors of the unmanned aerial vehicles with respect to the target position is proposed for antipodal tracking instead of using the relative phase angle difference. To consider a more realistic situation, inequality constraints are considered for collision avoidance between unmanned aerial vehicles and control input saturations using penalty functions in the model predictive control scheme. Simulations with a pair of unmanned aerial vehicles are done using a realistic car trajectory data in a urban environment in the United Kingdom to verify the feasibility and benefit of the proposed approach with comparing to a Lyapunov vector field guidance.

\footnotetext{
${ }^{*}$ This paper was presented at AIAA Guidance, Navigation, and Control Conference, Portland, Oregon, US, 8-11 Aug 2011 $\dagger$ Lecturer, Department of Informatics and Systems Engineering, AIAA Member; s.kim@cranfield.ac.uk.

$\ddagger$ PhD Student, Department of Informatics and Systems Engineering, AIAA Student Member; h.oh@cranfield.ac.uk.

§Professor, Department of Informatics and Systems Engineering, AIAA Member; a.tsourdos@cranfield.ac.uk.
} 


\section{Introduction}

In the last decade, using a swarm of UAV(Unmanned Airborne Vehicle)s has received attention to accomplish synergy with manned systems in both military and civil applications. Searching and subsequent tracking of moving ground target of interest is one of the important capabilities of UAVs required to increase an overall knowledge of the target's intent and to take proactive measures. In performing such missions, UAVs are to keep a certain distance from the moving target, known as a standoff distance, in order to track it without being noticed and at the same time to acquire the accurate target information. Lawrence ${ }^{1}$ firstly proposed the application of Lyapunov vector fields for standoff coordination of multiple UAVs, which was further investigated by Frew et. al. ${ }^{2-4}$ to include phase-keeping as well as standoff tracking. They invented a decoupled control structure in which speed and rate of heading change are separately controlled for standoff distance and phase angle keeping, respectively. Quigley et. al. ${ }^{5}$ firstly applied a concept of Hopf bifurcation $^{6}$ to standoff tracking which has faster convergence to the standoff circle than the Lyapunov vector field approach. Chen $\mathrm{el} . \quad a .^{7}$ proposed the use of a tangent-plus-Lyapunov vector field which includes a simple switching logic between tangent and Lyapunov vector fields to make convergence to the standoff circle faster. Wise and Rysdyk ${ }^{8}$ well surveyed and compared the different methodologies for standoff tracking: Helmsman behavior, Lyapunov vector field, controlled collective motion, and model predictive control. Recently, Prevost et. al. ${ }^{9}$ applied a receding horizon model-based predictive control for standoff tracking. Although the aforementioned two works tried to apply the model predictive control (also known as receding horizon control) to standoff tracking, Wise and $\mathrm{Rysdyk}^{8}$ used only a simple relative kinematics variation between UAV and target with still decoupling the speed and rate of heading change, and Prevost et. al. ${ }^{9}$ focused on a single UAV applications for only the standoff distance keeping with a simple UAV/target trajectory prediction.

Unlike the aforementioned works, this study aims at developing a NMPCST(Nonlinear Model Predictive Coordinated Standoff Tracking) framework for both standoff distance and phase angle keeping by a pair UAVs. The Lyapunov vector field, the Hopf bifurcation and so on ${ }^{2,4,5,8}$ used the heading control for standoff distance keeping and the speed control for phase keeping, separately. Differently from these decoupled controller structure of the previous works, the most valuable benefit of this approach is that it can get an optimal performance in terms of tracking accuracy and fuel consumption. In this way, the proposed optimal control formulation adds value by providing a basis for gain selection or parameter tuning. The overall controller structure of UAV formation considered in this study is fully decentralized in a fact that each UAV optimizes its controller based on the future state predictions of the pair UAV to cooperate with and the target to be tracked. This decentralized optimization is performed after receiving only the control at the previous sampling, the current state, and the current target state/covariance estimation of the pair 
UAV via communication. For target trajectory prediction, this paper uses an acceleration model for realistic target dynamics, which can consider reasonable system noise covariance matrix reflecting the target's motion characteristics. For antipodal standoff tracking, the phase keeping term is included in a performance index of optimization framework as well as standoff-distance keeping. To simplify optimization formulation and decrease computation burden, a new manipulation using inner product of position vectors of the UAVs with respect to the target position is proposed instead of using the relative phase angle difference which were used in most of the existing works. ${ }^{2,4,8}$ To accomplish the safety under realistic situations, inequality constraints are also considered for collision avoidance between UAV members and control input saturations in the model predictive control scheme. Lastly, simulations with a pair of UAVs are done using realistic car trajectory data in urban traffic in the UK to verify the feasibility and benefits of the proposed approach.

The overall structure of this paper is given as: Section II contains a definition of UAV dynamic model, ground target model, and sensor model considered in this study. Section III discusses the tracking filter design and the sensor fusion for target localization and prediction. As the main part of this paper, Section IV proposes the decentralized structure, definition of performance index and constraints, and nonlinear model predictive controller design for coordinated standoff tracking. Section V presents the simulation result using ground driving vehicle trajectory data acquired from a traffic simulation program compared with Lyapunov vector field guidance. Lastly, conclusions and future works are given in Section VI.

\section{Problem Definition}

\section{A. UAV Dynamic Model}

Assuming each UAV has a low-level flight controller such as SAS(Stability Augmentation System) and CAS(Controllability Augmentation System) for heading and velocity hold functions, this study aims to design the model-predictive guidance inputs to this low-level controller for coordinated standoff tracking. Consider a two-dimensional UAV kinematic model as:

$$
\left(\begin{array}{c}
\dot{x} \\
\dot{y} \\
\dot{\psi} \\
\dot{v} \\
\dot{\omega}
\end{array}\right)=f(\mathbf{x}, \mathbf{u})=\left(\begin{array}{c}
v \cos \psi \\
v \sin \psi \\
\omega \\
-\frac{1}{\tau_{v}} v+\frac{1}{\tau_{v}} u_{v} \\
-\frac{1}{\tau_{\omega}} \omega+\frac{1}{\tau_{\omega}} u_{\omega}
\end{array}\right)
$$

where $\mathbf{x}=(x, y, \psi, v, \omega)^{T}$ are the inertial position, heading, speed and yaw rate of the UAV, respectively. $\tau_{v}$ and $\tau_{\omega}$ are time constants for considering actuator delay. $\mathbf{u}=\left(u_{v}, u_{\omega}\right)^{T}$ are the commanded speed and 
turning rate constrained by the following dynamic limits of UAV:

$$
\begin{array}{r}
\left|u_{v}-v_{0}\right| \leq v_{\max } \\
\left|u_{\omega}\right| \leq \omega_{\max }
\end{array}
$$

where $v_{0}$ is a nominal speed of UAV. To design the nonlinear model predictive controller, the continuous UAV model in Eq. (1) can be discretized by Euler integration into:

$$
\mathbf{x}_{k+1}=f_{d}\left(\mathbf{x}_{k}, \mathbf{u}_{k}\right)=\mathbf{x}_{k}+T_{s} f\left(\mathbf{x}_{k}, \mathbf{u}_{k}\right)
$$

where $\mathbf{x}_{k}=\left(x_{k}, y_{k}, \psi_{k}, v_{k}, \omega_{k}\right)^{T}, \mathbf{u}_{k}=\left(u_{v k}, u_{\omega k}\right)^{T}$, and $T_{s}$ is a sampling time.

\section{B. Ground Target Model}

General target tracking filters have traditionally been developed for monitoring aerial targets such as airplanes, missiles and so on. Although ground vehicles move with much lower speeds than aerial targets, they often and irregularly perform stop-and-go maneuvers with much smaller turn radius. A constant velocity model broadly used for radar target tracking seems thus unsuitable for tracking ground vehicles, and hence an acceleration or a jerk model can be a candidate instead. After analyzing the car trajectory data acquired by running a S-Paramics traffic simulation program ${ }^{10}$ and general driving behaviors, it is observed that the jerk is not negligible, but the acceleration is piecewise constant for a specific duration of time. This study, therefore, considers acceleration dynamics ${ }^{11}$ to apply it to tracking of the moving ground vehicle. This acceleration model regards the target acceleration as a process correlated and exponentially decreasing in time, which means if there is a certain acceleration rate at a time $t$ then it is likely to be the same jerk also at a time instant $t+\tau$. Then, discretized system equations for acceleration model are expressed as:

$$
\mathbf{x}_{k+1}^{t}=F_{k} \mathbf{x}_{k}^{t}+\eta_{k}
$$


where $\mathbf{x}_{k}^{t}=\left(x_{k}^{t}, \dot{x}_{k}^{t}, \ddot{x}_{k}^{t}, y_{k}^{t}, \dot{y}_{k}^{t}, \ddot{y}_{k}^{t}\right)^{T}$, and $\eta_{k}$ is a process noise which represents the acceleration characteristics of the target. The state transition matrix $F_{k}$ can be represented as follows: ${ }^{11,12}$

$$
F_{k}=\left[\begin{array}{cccccc}
1 & T_{s} & \left(e^{-\alpha T_{s}}+\alpha T_{s}-1\right) / \alpha^{2} & 0 & 0 & 0 \\
0 & 1 & \left(1-e^{-\alpha T_{s}}\right) / \alpha & 0 & 0 & 0 \\
0 & 0 & e^{-\alpha T_{s}} & 0 & 0 & 0 \\
0 & 0 & 0 & 1 & T_{s} & \left(e^{-\alpha T_{s}}+\alpha T_{s}-1\right) / \alpha^{2} \\
0 & 0 & 0 & 0 & 1 & \left(1-e^{-\alpha T_{s}}\right) / \alpha \\
0 & 0 & 0 & 0 & 0 & e^{-\alpha T_{s}}
\end{array}\right]
$$

where $\alpha$ is a correlation parameter which allows for the modeling of the different classes of targets: small $\alpha$ for targets with relatively slow maneuvers and high $\alpha$ for targets with fast and evasive ones. The covariance matrix of the process noise $\eta_{k}$ can be modeled as follows:

$$
Q_{k}=V\left[\eta_{k}\right]=\frac{\sigma_{a}^{2}}{\alpha^{4}}\left[\begin{array}{cccccc}
q_{11} & q_{12} & q_{13} & 0 & 0 & 0 \\
q_{12} & q_{22} & q_{23} & 0 & 0 & 0 \\
q_{13} & q_{23} & q_{33} & 0 & 0 & 0 \\
0 & 0 & 0 & q_{11} & q_{12} & q_{13} \\
0 & 0 & 0 & q_{12} & q_{22} & q_{23} \\
0 & 0 & 0 & q_{13} & q_{23} & q_{33}
\end{array}\right]
$$

where $\sigma_{a}$ is the standard deviation related to target acceleration, and a definition of $q_{i j}$ is: ${ }^{11}$

$$
\begin{aligned}
& q_{11}=\left(1-l+2 m+2 / 3 m^{3}-2 m^{2}-4 m \sqrt{l}\right) \\
& q_{12}=\alpha\left(l+1-1 / l-2 m+m^{2}\right) \\
& q_{13}=\alpha^{2}(1-l-2 m \sqrt{l}) \\
& q_{22}=\alpha^{2}(4 \sqrt{l}-3-l+2 m) \\
& q_{23}=\alpha^{3}(l+1-2 \sqrt{l}) \\
& q_{33}=\alpha^{4}(1-l)
\end{aligned}
$$

where $l=e^{-2 \alpha T_{s}}$ and $m=\alpha T_{s}$. 


\section{Sensor Model of Moving Target Indicator Radar}

This study assumes the UAVs are equipped with a MTIR (Moving Target Indicator Radar) to localize the position of target. Because the measurement of MTIR is composed of range and azimuth of the target with respect to the radar location, the actual measurements is the relative range and azimuth with respect to the position of the airborne UAV. The radar measurement $(r, \phi)^{T}$ can be defined as the following nonlinear relationship using the target position $\left(x_{k}^{t}, y_{k}^{t}\right)^{T}$ and the UAV position $\left(x_{k}, y_{k}\right)^{T}$ as:

$$
\left(\begin{array}{c}
r_{k} \\
\phi_{k}
\end{array}\right)=h\left(\mathbf{x}_{k}^{t}\right)+\nu_{k}=\left(\begin{array}{c}
\sqrt{\left(x_{k}^{t}-x_{k}\right)^{2}+\left(y_{k}^{t}-y_{k}\right)^{2}} \\
\tan ^{-1} \frac{y_{k}^{t}-y_{k}}{x_{k}^{t}-x_{k}}
\end{array}\right)+\nu_{k}
$$

where $\tan ^{-1}$ is to be executed as the four quadrant inverse tangent in practice, $\nu_{k}$ is a measurement noise vector, and its noise covariance matrix is defined as:

$$
V\left[\nu_{k}\right]=R=\left[\begin{array}{cc}
\sigma_{r}^{2} & 0 \\
0 & \sigma_{\phi}^{2}
\end{array}\right]
$$

\section{Tracking Filter Design for Target Localization and Prediction}

\section{A. Extended Kalman Tracker}

Considering $h\left(\mathbf{x}_{k}^{t}\right)$ in the measurement equation is nonlinear as shown in Eq. (14), the localization of target can be designed using an EKF(Extend Kalman Filter) ${ }^{13,14}$ as:

\section{Time update}

$$
\begin{aligned}
& \mathbf{x}_{k \mid k-1}^{t}=F_{k} \mathbf{x}_{k-1 \mid k-1}^{t} \\
& P_{k \mid k-1}=F_{k} P_{k-1 \mid k-1} F_{k}^{T}+Q_{k}^{T}
\end{aligned}
$$

\section{Measurement update}

$$
\begin{aligned}
K & =P_{k \mid k-1} H_{k}^{T}\left\{H_{k} P_{k \mid k-1} H_{k}^{T}+R_{k}\right\}^{-1} \\
\mathbf{x}_{k \mid k}^{t} & =\mathbf{x}_{k \mid k-1}^{t}+K\left\{\mathbf{z}_{k}-h\left(\mathbf{x}_{k \mid k-1}^{t}\right)\right\} \\
P_{k \mid k} & =\left(I-K H_{k}\right) P_{k \mid k-1} .
\end{aligned}
$$


where $\mathbf{z}_{k}$ is a measurement vector. The output matrix $H_{k}$ is a Jacobian of Eq. (14) with respect to the time-update state $\mathbf{x}_{k \mid k-1}^{t}$ as

$$
H_{k}=\left.\frac{\partial h}{\partial \mathbf{x}_{k}^{t}}\right|_{\mathbf{x}_{k}^{t}=\mathbf{x}_{k \mid k-1}^{t}}
$$

where

$$
\frac{\partial h}{\partial \mathbf{x}_{k}^{t}}=\left[\begin{array}{cccccc}
\frac{x_{k}^{t}-x_{k}}{\sqrt{\left(x_{k}^{t}-x_{k}\right)^{2}+\left(y_{k}^{t}-y_{k}\right)^{2}}} & 0 & 0 & \frac{y_{k}^{t}-y_{k}}{\sqrt{\left(x_{k}^{t}-x_{k}\right)^{2}+\left(y_{k}^{t}-y_{k}\right)^{2}}} & 0 & 0 \\
-\cos ^{2}\left\{\tan ^{-1}\left(\frac{y_{k}^{t}-y_{k}}{x_{k}^{t}-x_{k}}\right)\right\} \frac{y_{k}^{t}-y_{k}}{\left(x_{k}^{t}-x_{k}\right)^{2}} & 0 & 0 & \cos ^{2}\left\{\tan ^{-1}\left(\frac{y_{k}^{t}-y_{k}}{x_{k}^{t}-x_{k}}\right)\right\} \frac{1}{x_{k}^{t}-x_{k}} & 0 & 0
\end{array}\right]
$$

\section{B. State-vector fusion}

Since this study assumes a pair of UAVs carry out the coordinated standoff tracking of a ground moving target, each UAV's MTIR sensor can get its own measurement and execute the tracking filter algorithm separately. After each UAV receives the other's estimation via communication link, it can run a decentralized sensor fusion to enhance the tracking accuracy. This study simply adopts the following state-vector fusion also known as a track-to-track fusion ${ }^{15}$ under the assumption that the communication bandwidth is wide enough to transmit the state (6-by-1) and covariance matrix (6-by-6) in both directions between a pair of UAVs.

$$
\begin{aligned}
\hat{\mathbf{x}}_{k}^{t} & =\mathbf{x}_{k \mid k}^{t}+P_{k \mid k}\left(P_{k \mid k}+P_{k \mid k}^{p}\right)^{-1}\left(\mathbf{x}_{k \mid k}^{t p}-\mathbf{x}_{k \mid k}^{t}\right) \\
P_{k} & =P_{k \mid k}+P_{k \mid k}\left(P_{k \mid k}+P_{k \mid k}^{p}\right)^{-1} P_{k \mid k}^{T}
\end{aligned}
$$

where $\mathbf{x}_{k \mid k}^{t p}$ and $P_{k \mid k}^{p}$ represent the state and error covariance estimations of the pair UAV. Instead of the state-vector fusion, the fusion using information filter ${ }^{16}$ can be used for minimizing the communication load. Subsequently, $\hat{\mathbf{x}}_{k}^{t}$ in Eq. (23) will be used as the initial value of the state for the model prediction of the target for nonlinear model predictive control at each sampling.

\section{Nonlinear Model Predictive coordinated Standoff Target Tracking}

\section{A. Definition of Performance Index and Constraints}

The geometry between the UAV, the pair UAV, and the ground target considered in this study is shown in Fig. 1. The basic aim of coordinated standoff target tracking is to maintain a distance $|\vec{r}|$ between the UAV and the target as well as a relative phase angle $\Delta \theta$ between UAVs to a desired value concurrently. Then, coordinated standoff target tracking can be formulated as a nonlinear model predictive control problem to 


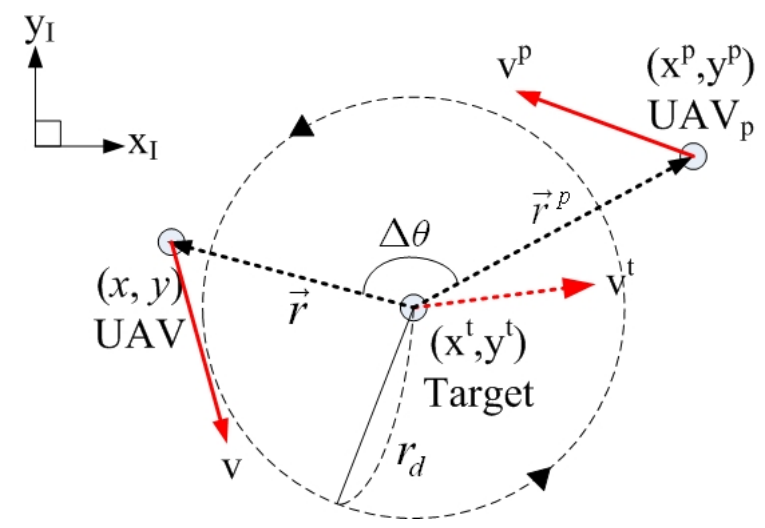

Figure 1. The geometry between the UAV, the pair UAV, and the ground target (The subscript $k$ is deleted for convenience.)

find a control input sequence $U_{k}=\left\{\mathbf{u}_{0}, \mathbf{u}_{1}, \ldots, \mathbf{u}_{N-1}\right\}$ that minimizes the following performance index.

$$
\begin{gathered}
J=\phi\left(\tilde{r}_{N}, \tilde{d}_{N}\right)+\sum_{k=0}^{N-1} L\left(\tilde{r}_{k}, \tilde{d}_{k}, \mathbf{u}_{\mathbf{k}}\right) \\
\phi\left(\tilde{r}_{N}, \tilde{d}_{N}\right)=\frac{1}{2}\left(p_{r} \tilde{r}_{N}^{2}+p_{d} \tilde{d}_{N}^{2}\right) \\
L\left(\tilde{r}_{k}, \tilde{d}_{k}, \mathbf{u}_{\mathbf{k}}\right)=\frac{1}{2}\left\{q_{r} \tilde{r}_{k}+q_{d} \tilde{d}_{k}^{2}+r_{v}\left(\frac{u_{v k}-v_{0}}{v_{\max }}\right)^{2}+r_{\omega}\left(\frac{u_{\omega k}-\frac{v_{0}}{r_{d}}}{\omega_{\max }}\right)^{2}\right\}
\end{gathered}
$$

where

$$
\begin{aligned}
\tilde{r}_{k} & =\frac{r_{d}^{2}-\left|\vec{r}_{k}\right|^{2}}{r_{d}^{2}} \\
\tilde{d}_{k} & =\frac{\vec{r}_{k}^{T} \vec{r}_{k}^{p}+\left|\vec{r}_{k}\right|\left|\vec{r}_{k}^{p}\right|}{r_{d}^{2}}
\end{aligned}
$$

with $\vec{r}_{k}=C \mathbf{x}_{k}-C_{t} \mathbf{x}_{k}^{t}$ and $\vec{r}_{k}^{p}=C \mathbf{x}_{k}^{p}-C_{t} \mathbf{x}_{k}^{t}$ representing the relative vectors from the target position to the positions of the current $\mathrm{UAV}$ and its pair UAV, respectively. $C \mathbf{x}_{k}, C \mathbf{x}_{k}^{p}$ and $C \mathbf{x}_{k}^{t}$ represent the positions of the current UAV, the pair UAV, and the ground target which will be propagated according to time update equation of the Extended Kalman tracker in Eq. (16), respectively. $C \in R^{2 \times 5}$ and $C_{t} \in R^{2 \times 6}$ is simply represented by the definition of state variables: $\mathbf{x}_{k}=\left(x_{k}, y_{k}, \psi_{k}, v_{k}, \omega_{k}\right)^{T}, \mathbf{x}_{k}^{p}=\left(x_{k}^{p}, y_{k}^{p}, \psi_{k}^{p}, v_{k}^{p}, \omega_{k}^{p}\right)^{T}$, and 
$\mathbf{x}_{k}^{t}=\left(x_{k}^{t}, \dot{x}_{k}^{t}, \ddot{x}_{k}^{t}, y_{k}^{t}, \dot{y}_{k}^{t}, \ddot{y}_{k}^{t}\right)^{T}:$

$$
\begin{aligned}
C & =\left[\begin{array}{lllll}
1 & 0 & 0 & 0 & 0 \\
0 & 1 & 0 & 0 & 0
\end{array}\right] \\
C_{t} & =\left[\begin{array}{llllll}
1 & 0 & 0 & 0 & 0 & 0 \\
0 & 0 & 0 & 1 & 0 & 0
\end{array}\right] .
\end{aligned}
$$

Also, $r_{d}$ is a desired standoff distance from the UAVs to the ground target position, $N$ is the length of receding horizon, $v_{0}$ is a nominal speed of UAVs, and $\frac{v_{0}}{r_{d}}$ is a nominal angular velocity. $p_{r}, p_{d}, q_{r}, q_{d}, r_{v}$, and $r_{\omega}$ are constant weighting scalars.

In Eq. (29), $\tilde{d}_{k}$ is derived from the inner product of $\vec{r}_{k}$ and $\vec{r}_{k}^{p}$ as:

$$
<\vec{r}_{k}, \vec{r}_{k}^{p}>=\vec{r}_{k}^{T} \vec{r}_{k}^{p}=\left|\vec{r}_{k}\right|\left|\vec{r}_{k}^{p}\right| \cos \triangle \theta_{k}
$$

where $\Delta \theta_{k}=\left|\theta_{k}^{p}-\theta_{k}\right|$ with the phase angles of UAV positions with respect to the current target location, $\theta_{k}=\cos ^{-1} \frac{\left\langle\vec{r}_{k}, \hat{i}>\right.}{\left|\vec{r}_{k}\right|}$ and $\theta_{k}^{p}=\cos ^{-1} \frac{\left\langle\vec{r}_{k}^{p}, \hat{i}>\right.}{\left|\vec{r}_{k}^{p}\right|}\left(\hat{i}\right.$ is a x-direction unit vector.). If the phase difference $\Delta \theta_{k}$ is ideally maintained as $\pi$ radian for antipodal tracking of a pair of UAVs, the above equation is rearranged since $\cos \pi=-1$ as:

$$
\vec{r}_{k}^{T} \vec{r}_{k}^{p}+\left|\vec{r}_{k}\right|\left|\vec{r}_{k}^{p}\right|=0
$$

Therefore, the left-hand side of the above equation can be set as $\tilde{d}_{k}$ in the performance index to be minimized for maintaining the phase angle between a pair of UAVs. The merit of using this inner product concept rather than $\tilde{\theta}_{k}=\triangle \theta_{k}-\pi$ is two fold: the first is that practically it can minimize angular manipulations which might bring about unnecessary switching and laborious logic development at the boundary of angle range $(-\pi, \pi)$. The other is the unit of the phase-keeping term becomes the same as the first term in Eqs. (26)-(27) contributing to maintaining a standoff distance from the target. This is important for accomplishing the fast convergence of the optimization algorithm and also for avoiding unnecessary tuning of weighting parameters.

The dynamics of the UAVs in Eq. (4) is expressed as an equality constraint as:

$$
f_{d}\left(\mathbf{x}_{k}, \mathbf{u}_{k}\right)-\mathbf{x}_{k+1}=0 .
$$

The collision avoidance requirement between the UAV members is adopted as an inequality constraints as 
well as admissible control input ranges described in Eq. (2)-(3).

$$
\begin{aligned}
S_{v}\left(\mathbf{u}_{k}\right) & =\frac{\left|u_{1 k}-v_{0}\right|-v_{\max }}{v_{\max }} \leq 0 \\
S_{\omega}\left(\mathbf{u}_{k}\right) & =\frac{\left|u_{2 k}\right|-\omega_{\max }}{\omega_{\max }} \leq 0 \\
S_{c}\left(\mathbf{x}_{k}\right) & =\frac{r_{c}-\left|C\left(\mathbf{x}_{k}-\mathbf{x}_{k}^{p}\right)\right|}{r_{c}} \leq 0
\end{aligned}
$$

where $r_{c}$ is a safe distance between the UAVs to prevent collision.

\section{B. Nonlinear Model Predictive Standoff Tracking Algorithm}

The augmented performance index is defined with incorporating the equality and inequality constraints discussed in the previous section as:

$J_{a}=\phi\left(\tilde{r}_{N}, \tilde{d}_{N}\right)+\sum_{k=0}^{N-1}\left[L\left(\tilde{r}_{k}, \tilde{d}_{k}, \mathbf{u}_{k}\right)+\lambda_{k+1}^{T}\left\{f_{d}\left(\mathbf{x}_{k}, \mathbf{u}_{k}\right)-\mathbf{x}_{k+1}\right\}+\frac{1}{2} \mu_{v} l_{v k} S_{v}^{2}\left(\mathbf{u}_{k}\right)+\frac{1}{2} \mu_{\omega} l_{\omega k} S_{\omega}^{2}\left(\mathbf{u}_{k}\right)+\frac{1}{2} \mu_{c} l_{c k} S_{c}^{2}\left(\mathbf{x}_{k}\right)\right]$

where $\lambda_{k}$ is a Lagrange multiplier, and $\mu_{v}, \mu_{\omega}$, and $\mu_{c}$ are penalty function parameters. $l_{v k}, l_{\omega k}$, and $l_{c k}$ are defined to avoid unnecessary computation for satisfying inequality constraints as: ${ }^{17,18}$

$$
l_{* k}=\left\{\begin{array}{cc}
0, & S_{*} \leq 0 \\
1, & S_{*}>0
\end{array} .\right.
$$

To facilitate the derivation of optimal control law, let us define a Hamiltonian as:

$$
M_{k} \triangleq L\left(\tilde{r}_{k}, \tilde{d}_{k}, \mathbf{u}_{k}\right)+\lambda_{k+1}^{T} f_{d}\left(\mathbf{x}_{k}, \mathbf{u}_{k}\right)+\frac{1}{2} \mu_{v} l_{v k} S_{v}^{2}\left(\mathbf{u}_{k}\right)+\frac{1}{2} \mu_{\omega} l_{\omega k} S_{\omega}^{2}\left(\mathbf{u}_{k}\right)+\frac{1}{2} \mu_{c} l_{c k} S_{c}^{2}\left(\mathbf{x}_{k}\right)
$$

The variation of the augmented performance index is represented as:

$$
d J_{a}=\left(\frac{\partial \phi\left(\tilde{r}_{N}, \tilde{d}_{N}\right)}{\partial \mathbf{x}_{N}}-\lambda_{N}^{T}\right) d \mathbf{x}_{N}+\sum_{k=1}^{N-1}\left[\left(\frac{\partial M_{k}}{\partial \mathbf{x}_{k}}-\lambda_{k}^{T}\right) d \mathbf{x}_{k}+\frac{\partial M_{k}}{\partial \mathbf{u}_{k}} d \mathbf{u}_{k}\right]+\frac{\partial M_{0}}{\partial \mathbf{x}_{0}} d \mathbf{x}_{0}+\frac{\partial M_{0}}{\partial \mathbf{u}_{0}} d \mathbf{u}_{0}
$$

By selecting the Lagrange multiplier as:

$$
\begin{aligned}
\lambda_{N}^{T} & =\frac{\partial \phi\left(\tilde{r}_{N}, \tilde{d}_{N}\right)}{\partial \mathbf{x}_{N}} \\
\lambda_{k}^{T} & =\frac{\partial M_{k}}{\partial \mathbf{x}_{k}} \text { for } k=\mathrm{N}-1, \ldots, 0,
\end{aligned}
$$


the variation of $J_{a}$ is changed to:

$$
d J_{a}=\sum_{k=0}^{N-1} \frac{\partial M_{k}}{\partial \mathbf{u}_{k}} d \mathbf{u}_{k}+\lambda_{0}^{T} d \mathbf{x}_{0} .
$$

The right-hand side of Eq. (42) is derived from Eq. (26) as:

$$
\frac{\partial \phi\left(\tilde{r}_{N}, \tilde{d}_{N}\right)}{\partial \mathbf{x}_{N}}=p_{r} \tilde{r}_{N} \frac{\partial \tilde{r}_{N}}{\partial \mathbf{x}_{N}}+p_{d} \tilde{d}_{N} \frac{\partial \tilde{d}_{N}}{\partial \mathbf{x}_{N}}
$$

The right-hand side of Eq. (43) is derived from Eq. (40) as:

$$
\frac{\partial M_{k}}{\partial \mathbf{x}_{k}}=q_{r} \tilde{r}_{k} \frac{\partial \tilde{r}_{k}}{\partial \mathbf{x}_{k}}+q_{d} \tilde{d}_{k} \frac{\partial \tilde{d}_{k}}{\partial \mathbf{x}_{k}}+\lambda_{k+1}^{T} \frac{\partial f_{d}\left(\mathbf{x}_{k}, \mathbf{u}_{k}\right)}{\partial \mathbf{x}_{k}}+\mu_{c} l_{c k} S_{c}\left(\mathbf{x}_{k}\right) \frac{\partial S_{c}\left(\mathbf{x}_{k}\right)}{\partial \mathbf{x}_{k}}
$$

Jacobians in Eqs. (45)-(46) are derived from the definitions discussed in the previous section.

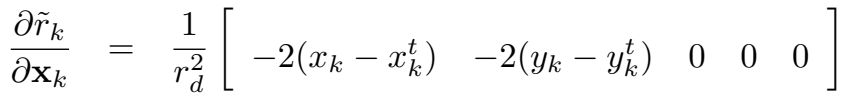

$$
\begin{aligned}
& \frac{\partial \tilde{d}_{k}}{\partial \mathbf{x}_{k}}=\frac{1}{r_{d}^{2}}\left[\begin{array}{lllll}
\left(x_{k}^{p}-x_{k}^{t}\right)+\frac{\left|\vec{r}_{k}^{p}\right|}{\left|\vec{r}_{k}\right|}\left(x_{k}-x_{k}^{t}\right) & \left(y_{k}^{p}-y_{k}^{t}\right)+\frac{\left|\vec{r}_{k}^{p}\right|}{\left|\vec{r}_{k}\right|}\left(y_{k}-y_{k}^{t}\right) & 0 & 0 & 0
\end{array}\right] \\
& \frac{\partial f_{d}\left(\mathbf{x}_{k}, \mathbf{u}_{k}\right)}{\partial \mathbf{x}_{k}}=\left[\begin{array}{ccccc}
1 & 0 & -T_{s} v_{k} \sin \psi_{k} & T_{s} \cos \psi_{k} & 0 \\
0 & 1 & T_{s} v_{k} \cos \psi_{k} & T_{s} \sin \psi_{k} & 0 \\
0 & 0 & 1 & 0 & T_{s} \\
0 & 0 & 0 & 1-\frac{1}{\tau_{v}} T_{s} & 0 \\
0 & 0 & 0 & 0 & 1-\frac{1}{\tau_{\omega}} T_{s}
\end{array}\right] \\
& \frac{\partial S_{c}\left(\mathbf{x}_{k}\right)}{\partial \mathbf{x}_{k}}=\frac{1}{r_{c}^{2}}\left[\begin{array}{lllll}
-\frac{x_{k}-x_{k}^{p}}{\left|\vec{r}_{k}-\vec{r}_{k}^{p}\right|} & -\frac{y_{k}-y_{k}^{p}}{\left|\vec{r}_{k}-\vec{r}_{k}^{p}\right|} & 0 & 0 & 0
\end{array}\right]
\end{aligned}
$$

Now substituting $d \mathbf{u}_{k}$ into Eq. (44) with the following equation which is basically a gradient method to minimize $M_{k}$

$$
d \mathbf{u}_{k}=-\Delta_{k} \frac{\partial M_{k}^{T}}{\partial \mathbf{u}_{k}}
$$

gives the following decreasing variation of $J_{a}$.

$$
d J_{a}=-\sum_{k=0}^{N-1} \Delta_{k} \frac{\partial M_{k}}{\partial \mathbf{u}_{k}} \frac{\partial M_{k}{ }^{T}}{\partial \mathbf{u}_{k}}+\lambda_{0}^{T} d \mathbf{x}_{0}
$$

Therefore, the control input can be updated using Eq. (51) as:

$$
\mathbf{u}_{k}^{i+1}=\mathbf{u}_{k}^{i}-\Delta_{k}{\frac{\partial M_{k}^{T}}{\partial \mathbf{u}_{k}}}^{T} \text { for } k=0, \ldots, \mathrm{N}-1
$$


where $i$ is the index of iteration, and $\Delta_{k}$ is a step size. $\frac{\partial M_{k}}{\partial \mathbf{u}_{k}}$ is defined as:

$$
\left(\frac{\partial M_{k}}{\partial \mathbf{u}_{k}}\right)^{T}=\left[\begin{array}{c}
\frac{r_{v}}{v_{\text {max }}^{2}}\left(u_{v k}-v_{0}\right) \\
\frac{r_{\omega}}{\omega_{\text {max }}^{2}}\left(u_{\omega k}-\frac{v_{0}}{r_{d}}\right)
\end{array}\right]+\lambda_{k+1}^{T} \frac{\partial f_{d}\left(\mathbf{x}_{k}, \mathbf{u}_{k}\right)}{\partial \mathbf{u}_{k}}+\left[\begin{array}{c}
\frac{\mu_{v}}{v_{\text {max }}^{2}} l_{v k} S_{v}\left(\mathbf{u}_{k}\right) \operatorname{sgn}\left(u_{v k}-v_{0}\right) \\
\frac{\mu_{\omega}}{\omega_{\text {max }}^{2}} l_{\omega k} S_{\omega}\left(\mathbf{u}_{k}\right) \operatorname{sgn}\left(u_{\omega k}-\frac{v_{0}}{r_{d}}\right)
\end{array}\right]
$$

where

$$
\frac{\partial f_{d}\left(\mathbf{x}_{k}, \mathbf{u}_{k}\right)}{\partial \mathbf{u}_{k}}=\left[\begin{array}{ccc}
1 & 0 \\
0 & 1 \\
0 & 0 \\
\frac{1}{\tau_{v}} T_{s} & 0 \\
0 & \frac{1}{\tau_{\omega}} T_{s}
\end{array}\right]
$$

and $\operatorname{sgn}(*)$ is a sign function satisfying

$$
\operatorname{sgn}(a)=\left\{\begin{array}{cc}
1 & \text { if } a \geq 0 \\
-1 & \text { if } a<0
\end{array} .\right.
$$

\section{Online Optimization via Decentralized Structure}

The online optimization is run under the decentralized structure at each sampling $T_{s}$ as shown in Fig. 2. When the MTIR measurement on the target composed of the relative range and azimuth with respect to each UAV position comes in, each UAV performs the localization of the target by the Extended Kalman tracker. Then, control $\left(U_{k-1}\right) /$ state $\left(\mathbf{x}_{k}\right)$ of the UAVs and those state $\left(\mathbf{x}_{k \mid k}^{t}\right) /$ covariance $\left(P_{k \mid k}\right)$ estimation information of the target are shared via communication between the UAVs. This study assumes all the required information can be shared between the UAVs via communications within a single sampling. If communications delay happens or optimizations are performed at slightly different instances between in a real world, the performance of the proposed approach might be degraded. However, our decentralized structure is advantageous in the sense that each UAV performs its own optimization individually using as best information as possible with a reduced computation burden compared to a centralized approach. Also, the proposed decentralized strategy can improve the probability of mission completion because the remaining pair UAV can continue the standoff tracking mission in spite of a failure of one UAV.

Then, each UAV carries out the state-vector fusion using its own estimation and the information from its pair UAV as shown in Eq. (23) in order to enhance the tracking accuracy against the moving target, which is then used as the initial value for propagating the target's state using the time update equation of 
the Extended Kalman tracker in Eq. (16). Following this, the state of the pair UAV is propagated using Eq. (4) with the received pair-UAV information: the current state information and the optimal control over the receding horizon computed at the previous sampling. Now all the external information is ready for the online optimization of nonlinear model predictive control.

The detailed online optimization has the following procedures: the initialization of control input over a receding horizon, state propagation (equality constraint), the computation of an augmented performance index, the finding of a Lagrange multiplier by backward integration, the computation of a Jacobian matrix of Hamiltonian $M_{k}$ with respect to the control input, and the update of control inputs. As a practical and efficient way, the initial guess of control inputs over the receding horizon at the first sampling $k=0$ can use a desired nominal speed of UAV and a provisional desired angular velocity as:

$$
U_{0}^{0}=\left\{\mathbf{u}_{0}, \mathbf{u}_{0}, \ldots, \mathbf{u}_{0}\right\} \in \Re^{2 \times N}
$$

where $\mathbf{u}_{0}=\left(u_{v 0}, u_{\omega 0}\right)^{T}, u_{v 0}=v_{d}$, and $u_{\omega 0}=\frac{v_{d}}{r}$. Then, if $k>0$, the initialization can be done by shifting the optimized control history from the previous sampling $U_{k-1}=\left\{\mathbf{u}_{0}, \mathbf{u}_{1}, \ldots, \mathbf{u}_{N-2}, \mathbf{u}_{N-1}\right\}$ as:

$$
U_{k}^{0}=\left\{\mathbf{u}_{1}, \mathbf{u}_{2}, \ldots, \mathbf{u}_{N-1}, \mathbf{u}_{N-1}\right\}
$$

The detailed optimization routine of the proposed approach is shown in Fig. 2. 
Figure 2. Decentralized Structure of Nonlinear Model Predictive coordinated Standoff Tracking

\begin{tabular}{|c|c|c|}
\hline current UAV & Communications & Pair UAV \\
\hline \multicolumn{3}{|l|}{ Start $k$-th sampling time } \\
\hline MTIR measurement acquisition & & MTIR measurement acquisition \\
\hline \multirow[t]{2}{*}{ EKF Target localization [Eqs.(16)-(20)] } & $\stackrel{\hat{\mathbf{x}}_{k \mid k}^{t p}, P_{k \mid k}^{p}}{\longrightarrow}$ & Target localization by EKF \\
\hline & $\overleftarrow{\hat{\mathbf{x}}_{k \mid k}^{t p}, P_{k \mid k}^{p}}$ & \\
\hline State-vector fusion [Eq.(23)] & & State-vector fusion \\
\hline \multirow[t]{2}{*}{ Target state propagation [Eq.(16)] } & $\stackrel{\mathbf{x}_{k}, U_{k-1}}{\longrightarrow}$ & Target state propagation \\
\hline & $\overleftarrow{\mathbf{x}_{k}^{p}, U_{k-1}^{p}}$ & \\
\hline Pair-UAV state propagation [Eq.(4)] & & Pair-UAV state propagation \\
\hline$i=1$ & & $i=1$ \\
\hline while $d J_{a}>\epsilon$ & & while $d J_{a}>\epsilon$ \\
\hline If $i==1$, & & If $i==1$, \\
\hline$U_{k}^{0}$ Initialization [Eqs.(57)-(58)] & & $U_{k}^{0}$ Initialization \\
\hline end & & end \\
\hline Self state propagation [Eq.(4)] & & Self state propagation \\
\hline \multicolumn{3}{|l|}{$J_{a}$ computation $[$ Eq. $(38)]$} \\
\hline$\lambda_{k}$ computation [Eqs.(42)-(43)] & & $:$ \\
\hline \multicolumn{3}{|l|}{ Computation of $\frac{\partial M_{k}}{\partial \mathbf{u}_{k}}[$ Eq.(54)] } \\
\hline If $d J_{a}<0: U_{k}^{i}=U_{k}^{i-1}-\Delta_{k} \frac{\partial M_{k}}{\partial \mathbf{u}_{k}}$ & & $\vdots$ \\
\hline \multicolumn{2}{|l|}{ else: reduce step size $\Delta_{k}$} & $:$ \\
\hline end & & end \\
\hline$i=i+1$ & & $i=i+1$ \\
\hline end & & end \\
\hline Start $k+1$-th sampling time & & \\
\hline
\end{tabular}




\section{Simulation result}

\section{A. Comparison to the Lyapunov vector field guidance method}

The guidance law to be compared is a LVFG(Lyapunov Vector Field Guidance) which was initially proposed by Lawrence ${ }^{1}$ and further developed by Frew et. al. ${ }^{2,4}$ The LVFG uses the following desired velocity $\left[\dot{x}_{d}, \dot{y}_{d}\right]^{T}$ (Hereafter the subscript $k$ will be omitted for simple notation.):

$$
\left[\begin{array}{c}
\dot{x}_{d} \\
\dot{y}_{d}
\end{array}\right]=\frac{-v_{d}}{r\left(r^{2}+r_{d}^{2}\right)}\left[\begin{array}{c}
\delta x\left(r^{2}-r_{d}^{2}\right)+\delta y\left(2 r r_{d}\right) \\
\delta y\left(r^{2}-r_{d}^{2}\right)-\delta x\left(2 r r_{d}\right)
\end{array}\right]
$$

where $r=\sqrt{\delta x^{2}+\delta y^{2}}=\sqrt{\left(x-x_{t}\right)^{2}+\left(y-y_{t}\right)^{2}}$ is the distance of the UAV from the ground vehicle. Herein $\left(x_{t}, y_{t}\right)$ is the position of the ground vehicle which can be estimated from the tracking filter, and $r_{d}$ is a desired standoff distance from the UAV to the ground vehicle. $v_{d}$ is a desired UAV speed. The desired heading can be decided using the desired two dimensional velocity components in Eq. (59) as:

$$
\psi_{d}=\tan ^{-1} \frac{\dot{y}_{d}}{\dot{x}_{d}}
$$

where $\tan ^{-1}$ is to be executed as the four quadrant inverse tangent in practice. The guidance command $u_{\omega}$ for turn rate is selected as the sum of proportional feedback and feedforward terms as:

$$
u_{\omega}=-k_{\omega}\left(\psi-\psi_{d}\right)+\dot{\psi}_{d}
$$

where

$$
\dot{\psi}_{d}=4 v_{d} \frac{r_{d} r^{2}}{\left(r^{2}+r_{d}^{2}\right)^{2}}
$$

If the velocity of a ground target vehicle could be estimated, the guidance vector needs to be adjusted in order to take a target velocity into account. Let us define the following relation between the new desired velocity of the UAV $\left[\dot{x}_{d n}, \dot{y}_{d n}\right]^{T}$ and the velocity of the ground vehicle $\left[\dot{x}_{t}, \dot{y}_{t}\right]$ using a scale factor $\alpha_{s}$ and the desired $x$ and $y$ velocity components derived in Eq. (59). ${ }^{2,4}$

$$
\left[\begin{array}{c}
\dot{x}_{d n} \\
\dot{y}_{d n}
\end{array}\right]=\left[\begin{array}{c}
\dot{x}_{t}+\alpha_{s} \dot{x}_{d} \\
\dot{y}_{t}+\alpha_{s} \dot{y}_{d}
\end{array}\right]
$$


The condition so that the UAV flies with the desired speed $V_{d}$ can be expressed by taking the norm of Eq. (63) as: $:^{2,4}$

$$
\left(\dot{x}_{d}^{2}+\dot{y}_{d}^{2}\right) \alpha_{s}^{2}+2\left(\dot{x}_{d} \dot{x}_{t}+\dot{y}_{d} \dot{y}_{t}\right) \alpha_{s}+\left(\dot{x}_{t}^{2}+\dot{y}_{t}^{2}-v_{d}^{2}\right)=0
$$

Eq. (64) has one positive real solution for $\alpha_{s}$ only if the desired speed of the UAV is larger than the target speed. $^{2}$ Substituting this solution into Eq. (63) yields the modified desired guidance vector of the UAV. Then the final modified desired heading can also be obtained using the relation $\psi_{d}=\tan ^{-1}\left(\dot{y}_{d n} / \dot{x}_{d n}\right)$.

The phase-angle keeping is separately accomplished by controlling the speed of UAVs as:

$$
\begin{aligned}
& u_{v}=k_{v}\left(\Delta \theta-\theta_{d}\right) r_{d}+v_{d} \\
& u_{v}^{p}=-k_{v}\left(\Delta \theta-\theta_{d}\right) r_{d}+v_{d}
\end{aligned}
$$

where $\theta$ is the azimuth angle of the UAVs relative to the target position, and $\theta_{d}=\pi$ is a desired phase difference between the UAVs.

\section{B. Assumptions and conditions}

The vehicle trajectory data acquired at $2 \mathrm{~Hz}$ in a S-Paramics ${ }^{10}$ traffic model of Devizes, Wiltshire, United Kingdom, are used to generate the MTIR measurements composed of relative range and azimuth angle with respect to a position of UAV as shown in Figure 3. The ground vehicle departs at the western side of Devizes

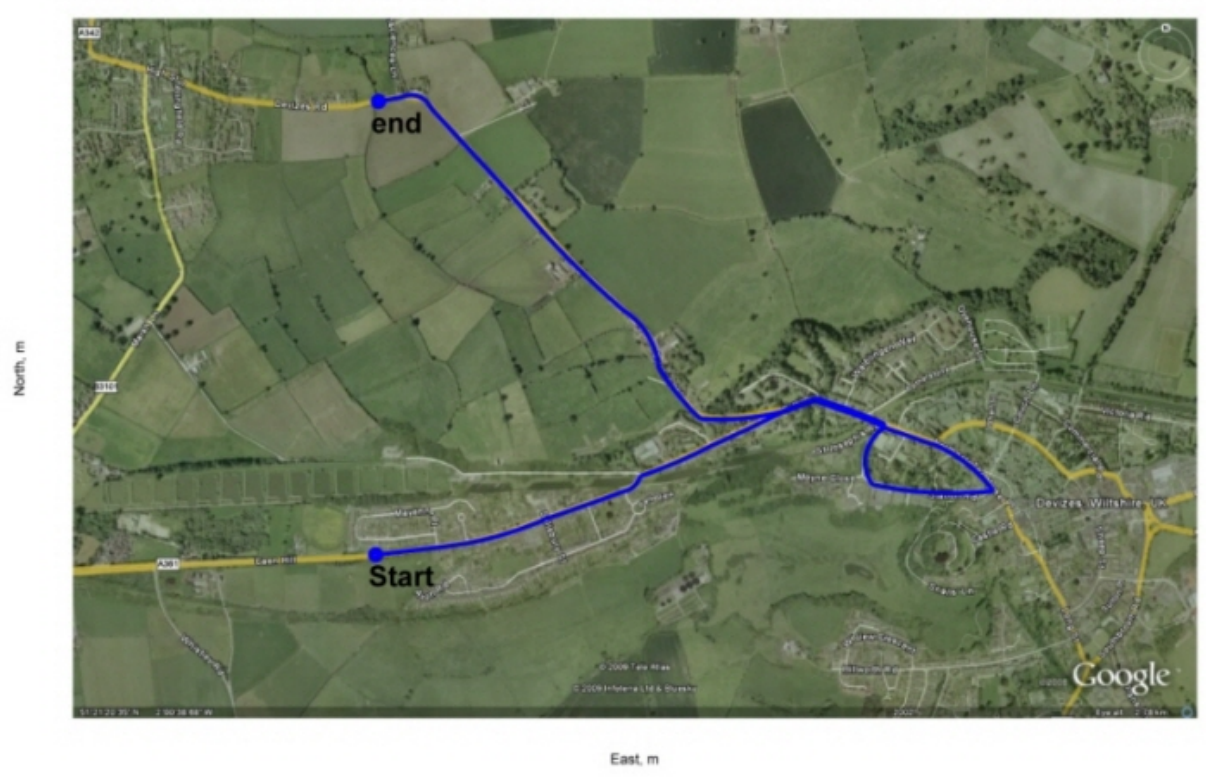

Figure 3. Ground Vehicle trajectory within the road network of Devizes, Wiltshire, United Kingdom with GIS satellite data overlaid thanks to Google Map 
and traverses a part of the town center and then turns back until the journey ends at the northwestern side of Devizes. As shown in Figs. 3-4, the ground vehicle moves complicatedly with frequently changing direction of driving, and the most challenge is its speed is irregularly changing. These true MTIR measurements of a pair of UAVs were mixed with the white noise having the following standard deviations.

$$
\begin{aligned}
& \text { UAV1: } \quad\left(\sigma_{r}, \sigma_{\phi}\right)=(5.0 \mathrm{~m}, 3.5 \mathrm{deg}) \\
& \text { UAV2: } \quad\left(\sigma_{r}, \sigma_{\phi}\right)=(7.5 \mathrm{~m}, 2.0 \mathrm{deg})
\end{aligned}
$$

The other setting of parameters needed for the NMPCST(Nonlinear Model Predictive Coordinated Standoff Tracking) and LVFG can be found in Table 1.

Table 1. Simulation parameters

\begin{tabular}{llc} 
Parameter & Value & Unit \\
\hline$T_{s}$ & 0.5 & $\mathrm{sec}$ \\
$\alpha$ & 0.6 & $\mathrm{~N} / \mathrm{A}$ \\
$\sigma_{a}$ & 0.66 & $\mathrm{~m} / \mathrm{s}^{2}$ \\
$\theta_{d}$ & $\pi$ & $\mathrm{rad}$ \\
$v_{0}, v_{d}$ & 40 & $\mathrm{~m} / \mathrm{s}$ \\
$r_{d}$ & 500 & $\mathrm{~m}$ \\
$r_{c}$ & 30 & $\mathrm{~m}$ \\
$v_{\max }$ & 10 & $\mathrm{~m} / \mathrm{s}$ \\
$\omega_{\max }$ & 0.2 & $\mathrm{rad} / \mathrm{s}$ \\
$\tau_{v}, \tau_{\omega}$ & $1 / 3$ & $\mathrm{sec}$ \\
$N$ & $4($ equivalent to $2 \mathrm{secs})$ & $\mathrm{N} / \mathrm{A}$ \\
$\left(p_{r}, p_{d}, q_{r}, q_{d}, r_{v}, r_{\omega}\right)$ & $\left(2 e 5,5 e 6, p_{r} / N, p_{d} / N, 1 e 2,5 e 1\right)$ & $\mathrm{N} / \mathrm{A}$ \\
$\mu_{v}, \mu_{\omega}, \mu_{c}$ & $1 e 3$ & $\mathrm{~N} / \mathrm{A}$ \\
Initial value of $\Delta_{k}$ & 0.3 & $\mathrm{~N} / \mathrm{A}$ \\
$\epsilon$ & 0.8 & $\mathrm{~N} / \mathrm{A}$ \\
$\left(k_{v}, k_{\omega}\right)$ & $(0.12,2)$ & $\mathrm{N} / \mathrm{A}$
\end{tabular}

\section{Simulation results}

To verify the feasibility and benefits of the proposed approach in this study, the same scenarios discussed in the previous section were fairly tested for both the LVFG and NMPCST. Firstly, the target localization by the extended Kalman filter and decentralized sensor fusion shows a reasonable accuracy even in $\mathrm{x}$ and y coordinate velocities as shown in Fig. 4. Figure 5 displays the absolute trajectories of UAVs and ground vehicle, and Figure 6 displays the relative trajectories of the UAVs with respect to the ground vehicle.

As can be seen in Figs. 6-7 and Table 2, the mean error of standoff-distance keeping by the NMPCST is much more lower than that of LVFG (which was tuned at best by trial and error procedure) even though 
its control consumption on rate of heading change is slightly higher than that of LVFG. This results from the fact that the NMPCST optimizes its control input by taking account of the propagated histories of the states of target and UAVs over the receding horizon. The control consumptions of $u_{v}$ and $u_{\omega}$ in Table 2 are computed by integrating the time histories of $\left|u_{v}-v_{0}\right|$ and $\left|u_{\omega}\right|$, respectively.

Although the mean error of phase-angle keeping by the LVFG could be improved by increasing the gain $k_{v}$, its value is set as 0.12 so that the LVFG has a similar level of control input consumption for fair comparison. As can be seen in Table 2, the NMPCST has a slightly lower mean error for phase-angle keeping than the LVFG with a similar level of speed consumption. This is beneficial to fixed-wing UAVs since they generally consume higher fuel when controlling their forward speed. Intuitively, this merit results from the fact that part of heading rate control as well as speed change by the NMPCST contributes on keeping a phase difference to meet the inner product relation of position vectors as in Eq. (33) during optimization. Figures 9-10 shows the control input histories of speed and heading-rate change of both the approaches.

The comparison performed in this simulation does not assert that the NMPCST can always get more optimal performance than the LVFG in terms of mission performance or fuel efficiency because the LVFG does not aim only precision in tracking. However, this simulation results implies that the proposed nonlinear model predictive approach can add a beneficial value to the coordinated standoff tracking problem by providing a basis for gain selection or parameter tuning. The mean computation time of each UAV's NMPCST routine at every sampling is approximately 0.1 second when using Intel Xeon CPU E5440 2.83GHz and 3GB RAM.

Table 2. Performance comparison between LVFG and NMPCST

\begin{tabular}{lcc} 
Parameter & LVFG & NMPCST \\
\hline mean standoff distance error $(\mathrm{m})$ & 25.73 & 7.87 \\
mean phase keeping error $(\mathrm{deg})$ & 7.61 & 7.28 \\
$u_{v}$ control consumption $(\mathrm{m} / \mathrm{s})$ & $18.79 \mathrm{e} 2$ & $17.33 \mathrm{e} 2$ \\
$u_{\omega}$ control consumption $(\mathrm{rad} / \mathrm{s})$ & 28.61 & 29.30
\end{tabular}

\section{Conclusions}

This paper proposed a novel nonlinear model predictive control framework for coordinated standoff tracking by a pair of unmanned aerial vehicles. The benefits of this approach are to add value to the coordinated standoff tracking problem by providing a guideline for gain selection or the trade-off of standoff tracking requirements. This can contribute towards acquiring optimal performance in terms of standoff tracking performance and fuel consumption compared to using the existing decoupled guidance structure. Also, this paper proposed the use of inner product manipulation for phase angle keeping, which helped 


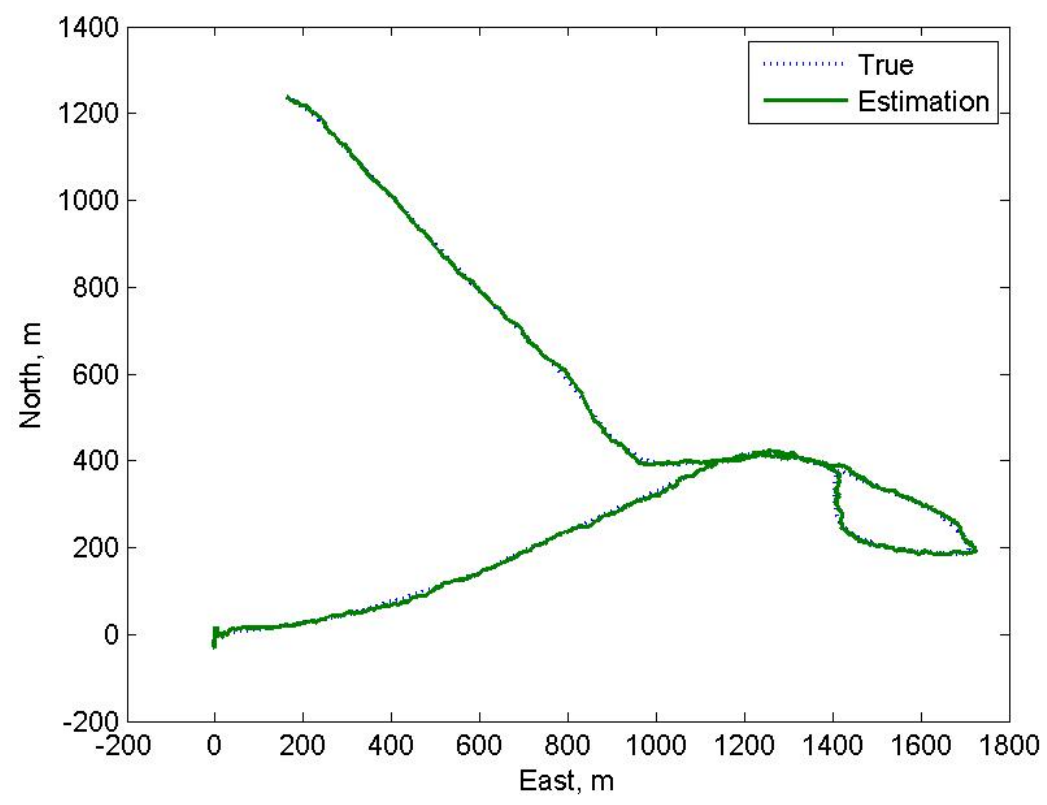

(a) Trajectory
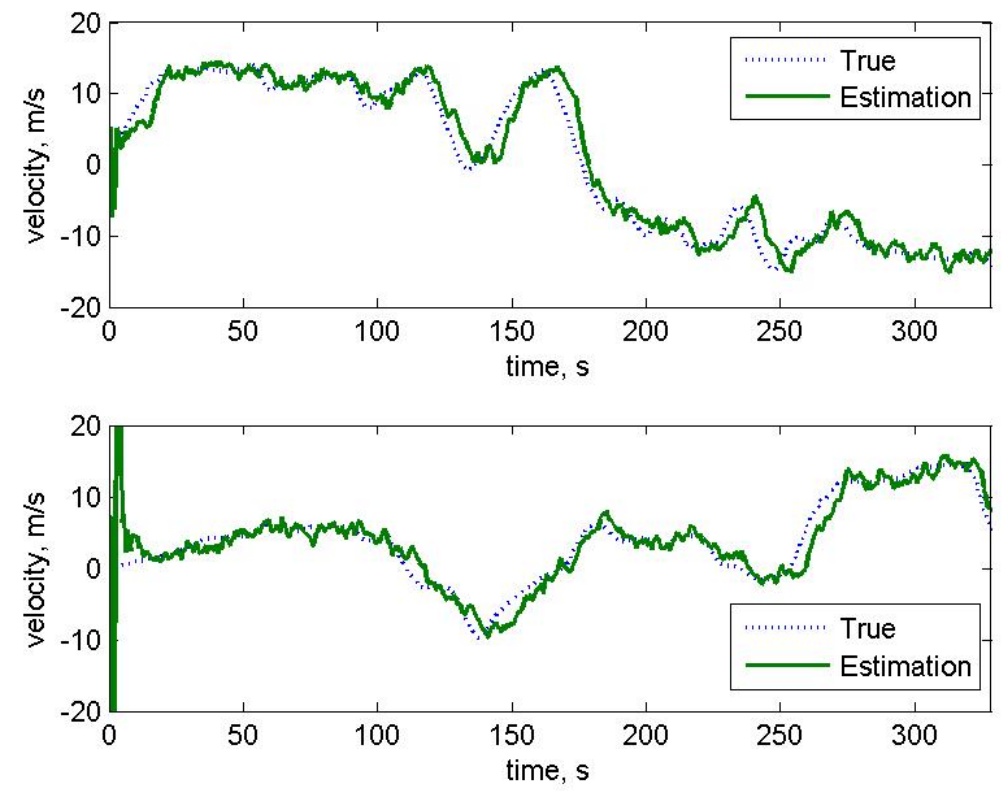

(b) $\mathrm{x}$ and y velocity

Figure 4. Ground target trajectory and velocity estimation using state-vector fusion based on the EKF 


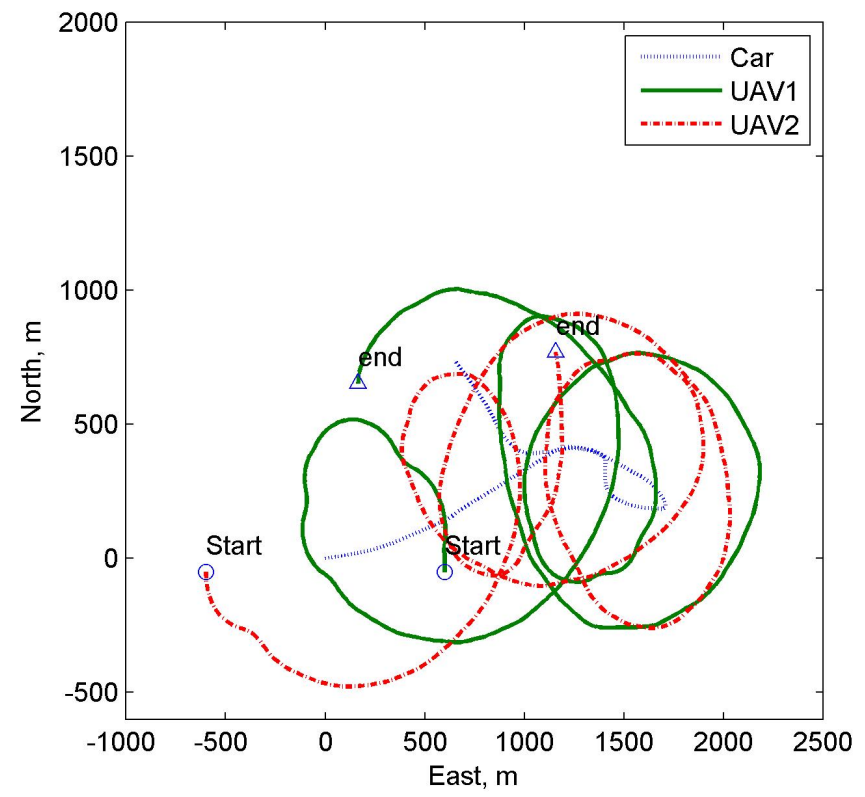

Figure 5. Absolute trajectories of UAVs using NMPCST and ground vehicle

minimize angular manipulation and weight tuning. Comparative simulations with a pair of unmanned aerial vehicles using a realistic car trajectory data in an urban environment were performed to show the properties and performance of the proposed nonlinear model predictive coordinated standoff tracking. One of the future works is to analyze the effect of changing the receding horizon size and to test different optimization solvers for standoff tracking problems. Then, systematic formulation of an automatic weight tuning will be another future work for avoiding the slow convergence when the UAVs locate far from the target initially. An additional important future work is to integrate the energy efficiency of UAVs into the performance index for optimization after mathematically formulating the relation between maneuver and fuel consumption of their engine or battery. The last promising future work is to integrate a map-based tracking filter with this model predictive scheme since it can provide more accurate target trajectory prediction. Since the proposed nonlinear model predictive coordinated standoff tracking is inherently decentralized, it can be easily extended to an unmanned system formation having more than two members for ground convoy and covert tracking.

\section{References}

${ }^{1}$ Lawrence, D., "Lyapunov vector fields for UAV flock coordination," 2nd AIAA Unmanned Unlimited conference, workshop, and exhibit, Reston, VA, USA, 2003.

${ }^{2}$ Frew, E., Lawrence, D., and Morris, S., "Coordinated standoff tracking of moving targets using Lyapunov guidance vector fields," Journal of guidance, control, and dynamics, Vol. 31, No. 2, 2008, pp. 290-306.

${ }^{3}$ Lawrence, D., Frew, E., and Pisano, W., "Lyapunov Vector Fields for Autonomous UAV Flight Control," Journal of 


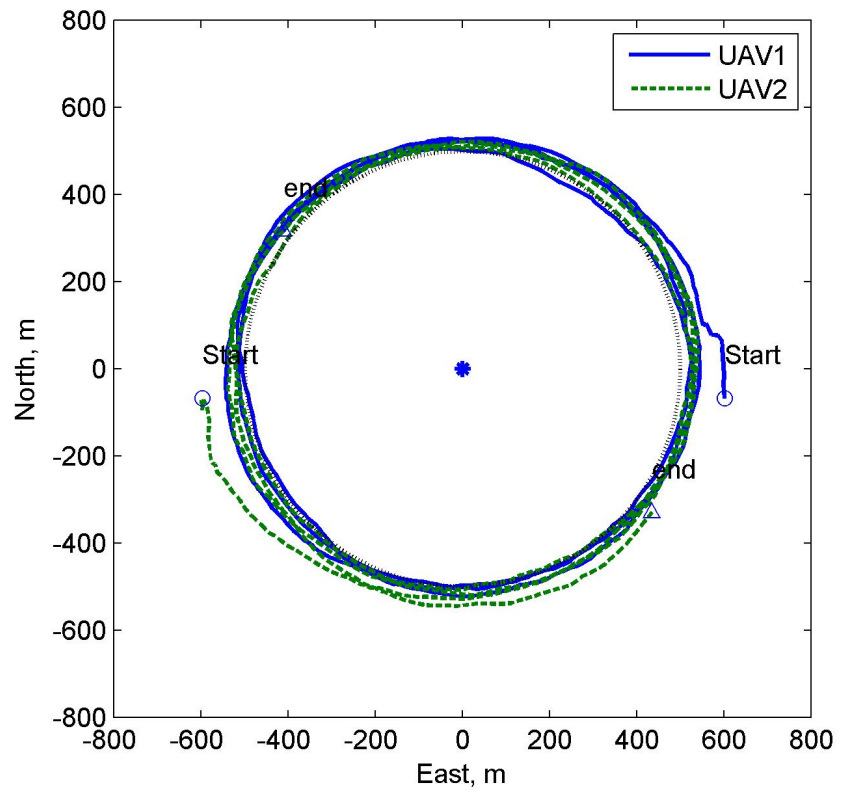

(a) LVFG

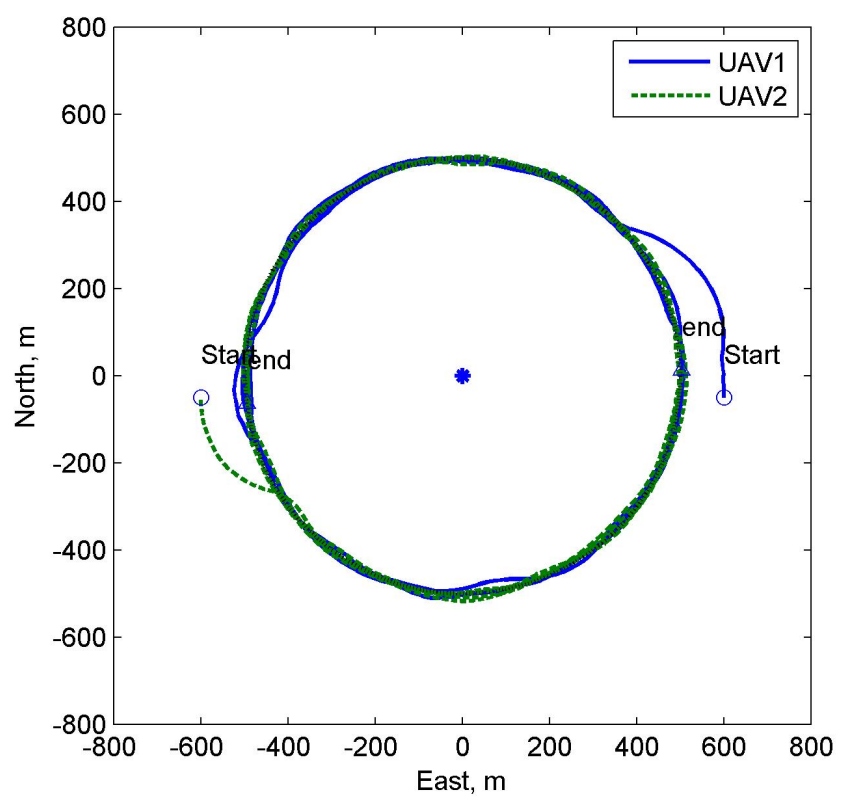

(b) NMPCST

Figure 6. Relative trajectories of UAVs with respect to ground vehicle 


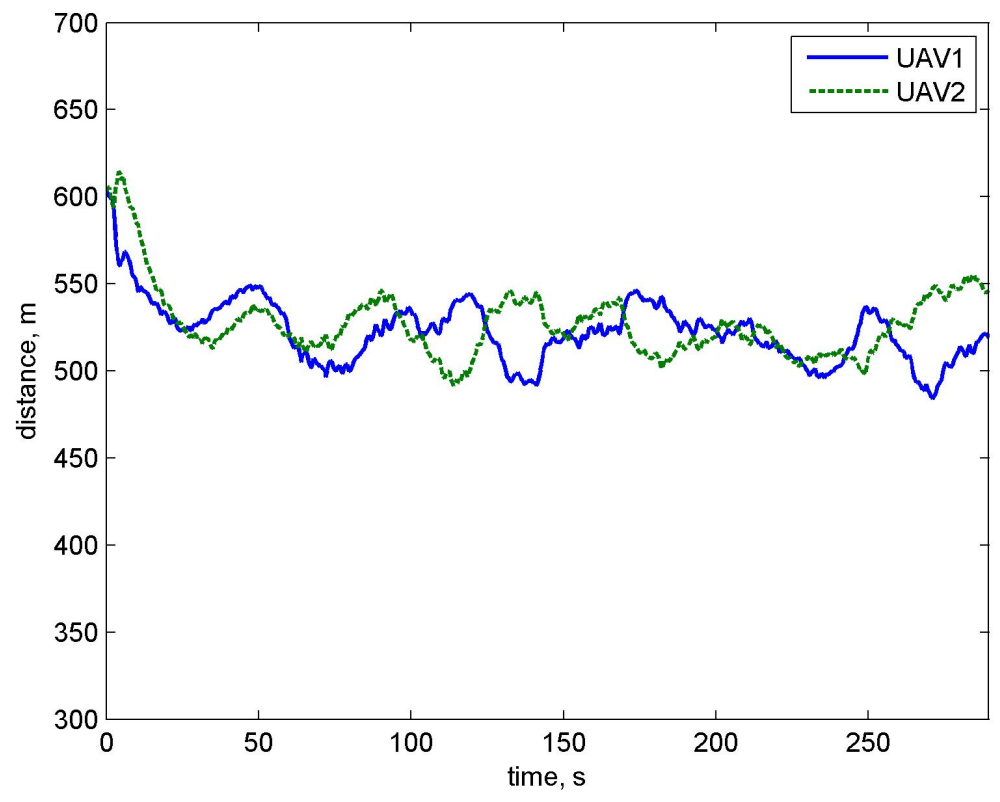

(a) LVFG

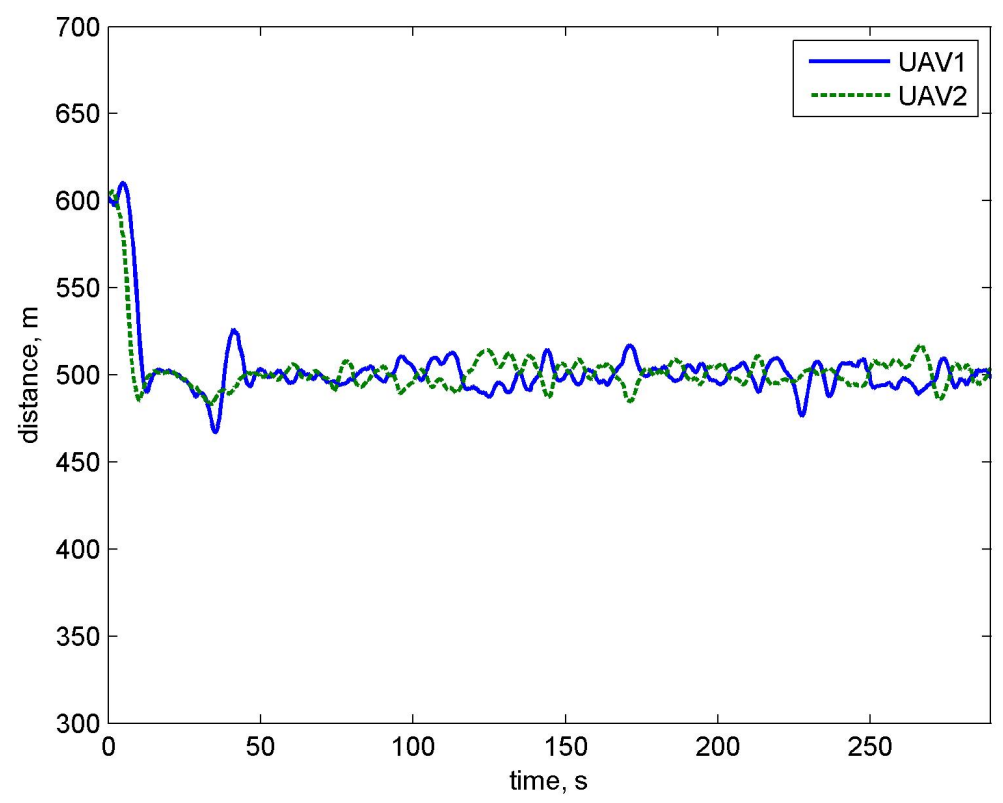

(b) NMPCST

Figure 7. Standoff distance from UAVs to ground vehicle 


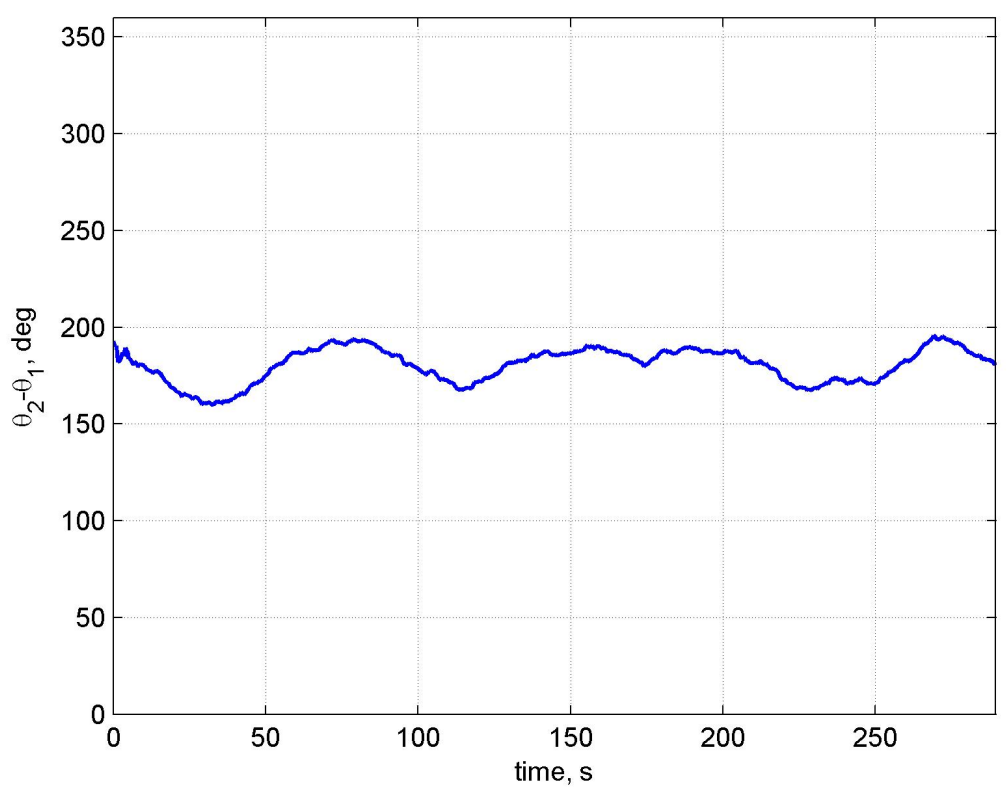

(a) LVFG

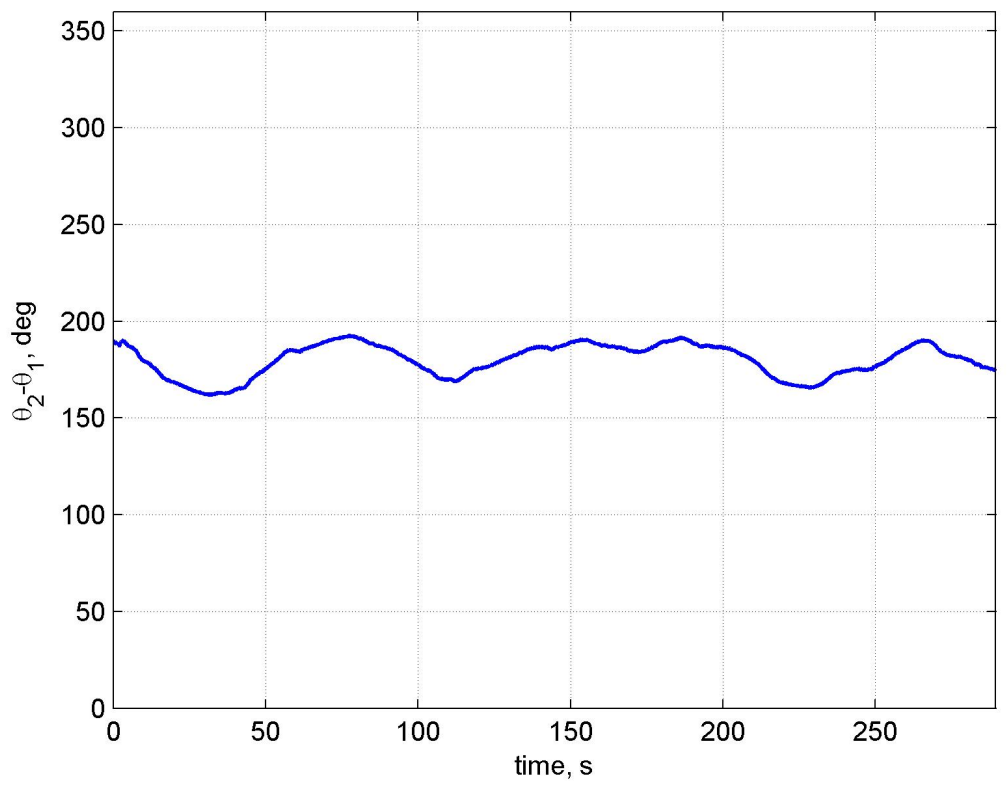

(b) NMPCST

Figure 8. Phase angle difference of UAVs with respect to ground vehicle 


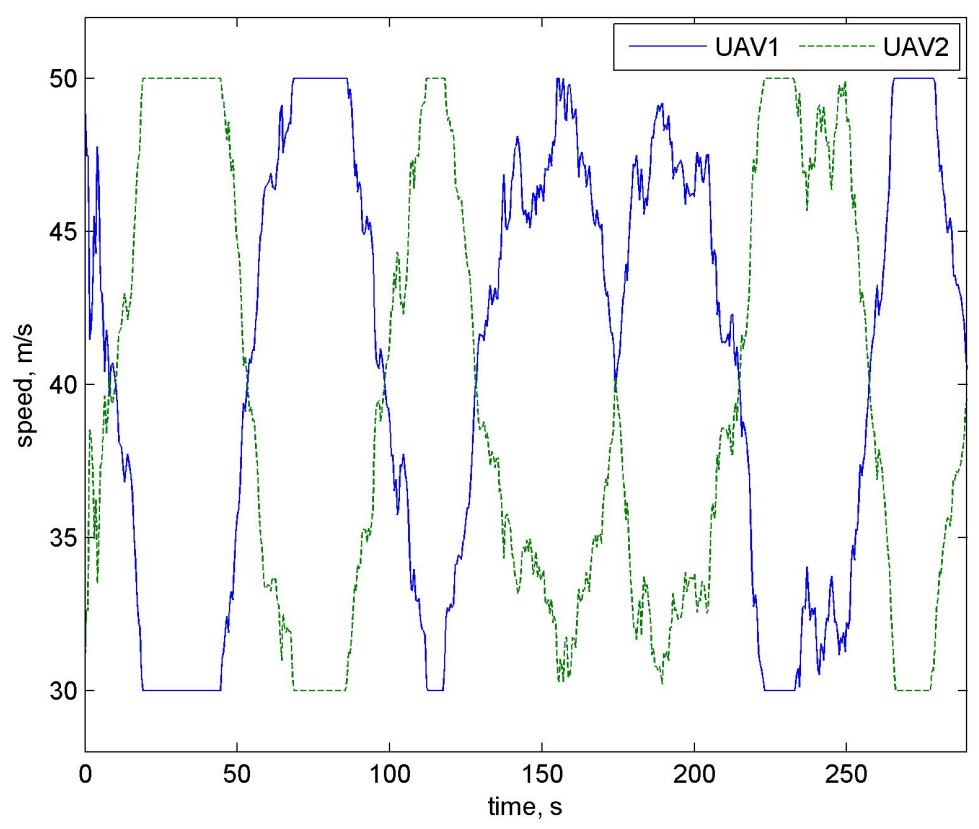

(a) LVFG

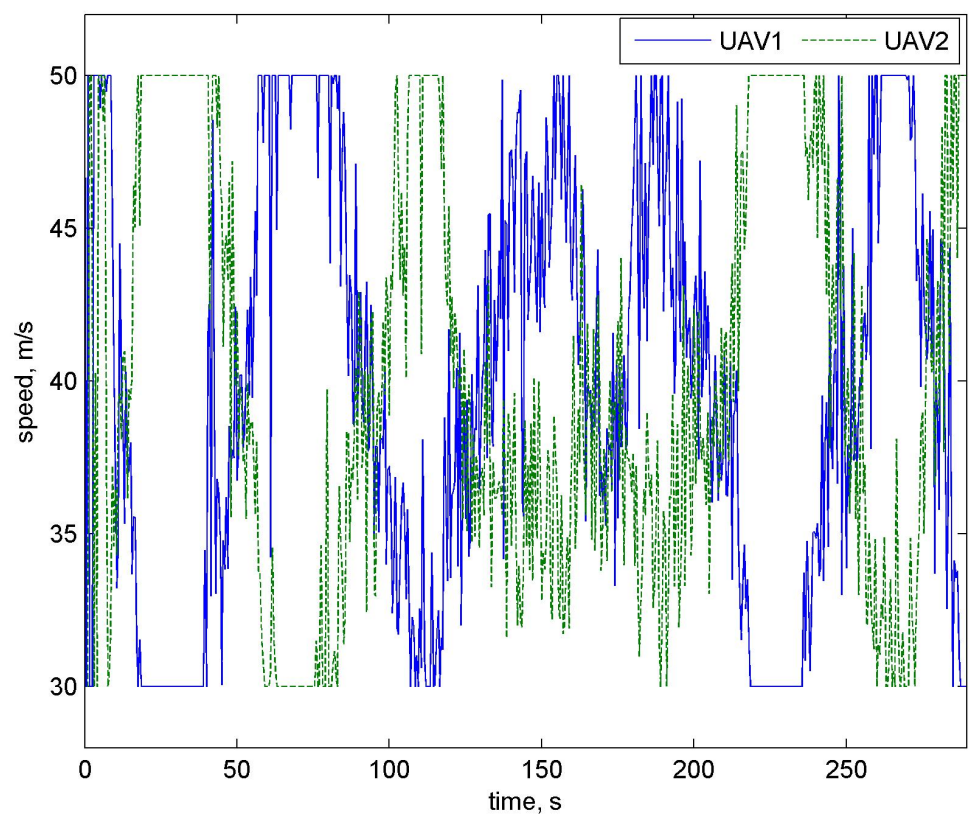

(b) NMPCST

Figure 9. Control input of UAVs: $u_{v}$ 


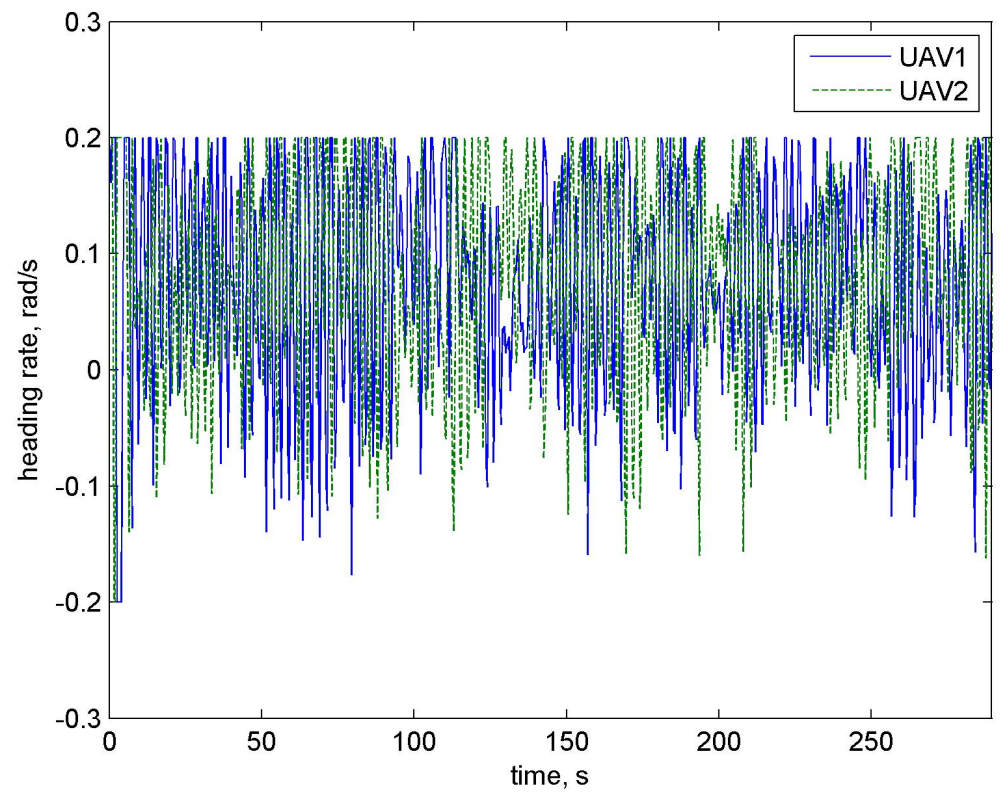

(a) LVFG

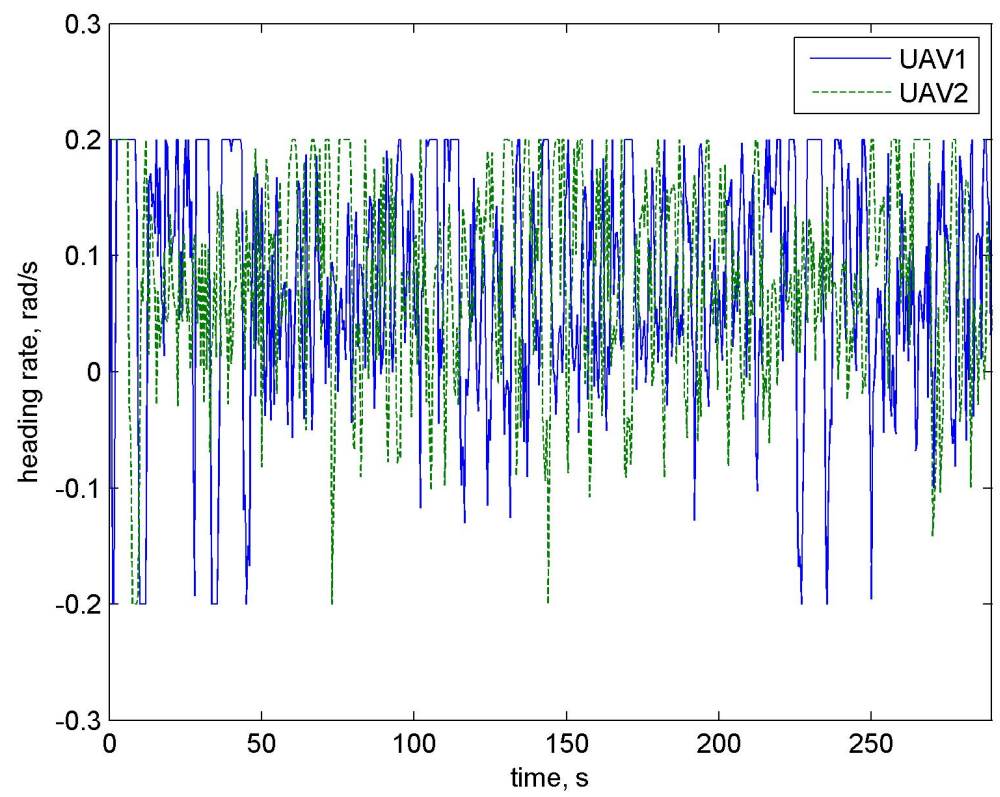

(b) NMPCST

Figure 10. Control input of UAVs: $u_{\omega}$ 
guidance, control, and dynamics, Vol. 31, No. 5, 2008, pp. 1220-1229.

${ }^{4}$ Morris, S. and Frew, E., "Cooperative tracking of moving targets by teams of autonomous unmanned air vehicles," Tech. Rep. FA9550-04-C-0107, MLB Company, 2005.

${ }^{5}$ Quigley, M., Goodrich, M., Griffiths, S., Eldredge, A., and Beard, R., "Target acquisition, localisation, and surveillance using a fixed-wing mini-UAV and gimbaled camera," IEEE(Institute of Electrical and Electronics Engineers) international conference on robotics and automation, Barcelona, Spain, 2005.

${ }^{6}$ Marsden, J. and McCracken, M., The Hopf Bifurcation and its applications, Vol 19 of Applied Mathematical Sciences, chap. 3, Springer-Verlag, 1976.

${ }^{7}$ Chen, H., Chang, K., and Agate, C., "Tracking with UAV using tanget-plus-lyapunov vector field guidance," International Conference on Information Fusion, Seattle, WA, USA, 2009.

${ }^{8}$ Wise, R. and Rysdyk, R., "UAV coordination for autonomous target tracking," AIAA Guidance, Navigation and Control Conference, Keystone, Colorado, USA, 2006.

${ }^{9}$ Prevost, C., Theriault, O., Desbiens, A., and Poulin, E., "Receding horizon model-based predictive control for dynamic target tracking: a comparative study," AIAA Guidance, Navigation, and Control Conference, Chicago, Illinois, USA, 2009.

${ }^{10}$ SIAS Limited, "S-Paramics Software," http://www.sias.com, Jan 2011.

${ }^{11}$ Mehrotra, K. and Mahapatra, P. R., "A jerk model for tracking highly maneuvering targets," IEEE(Institute of Electrical and Electronics Engineers) Transactions on Aerospace and Electronic Systems, Vol. 33, No. 4, 1997, pp. $1094-1105$.

${ }^{12}$ Kim, S., Zbikowski, R., Tsourdos, A., and White, B., "Behaviour Recognition of Ground Vehicle for Airborne Monitoring by UAV Swarm," IFAC(International Federation of Automatic Control) Symposium on Intelligent Autonomous Systems, Lecce, Italy, 2010.

${ }^{13}$ Haykin, S. et al., Kalman filtering and neural networks, chap. 5.2, Wiley Online Library, 2001.

${ }^{14}$ Blackman, S. and Popoli, R., Design and analysis of modern tracking systems, chap. 3.4-3.5, Artech House Noorwood, MA, 1999.

${ }^{15}$ Blackman, S. and Popoli, R., Design and analysis of modern tracking systems, chap. 10.4, Artech House Noorwood, MA, 1999.

${ }^{16}$ Mutambara, A., Decentralized Estimation and Control for Multisensor Systems, chap. 3-4, CRC Press LLC, Boca Raton, Florida, 1998.

${ }^{17}$ Shin, J. and Kim, H., "Nonlinear model predictive formation flight," IEEE(Institute of Electrical and Electronics Engineers) Transactions on Systems, Man and Cybernetics, Part A: Systems and Humans, Vol. 39, No. 5, 2009, pp. 1116-1125.

${ }^{18}$ Shin, H., Tahk, M., and Kim, H., "Nonlinear Model Predictive Control for Multiple UAVs Formation Using Passive Sensing," International Journal of Aeronautical and Space Sciences, Vol. 12, No. 1, 2011, pp. 16-23. 